\title{
Phylogenetic Assignment of Ralstonia pseudosolanacearum (Ralstonia solanacearum Phylotype I) Isolated from Rosa spp.
}

\author{
M. Bergsma-Vlami, ${ }^{\dagger}$ J. L. J. van de Bilt, N. N. A. Tjou-Tam-Sin, and M. Westenberg, Dutch National Plant Protection Organization \\ (NPPO-NL), P.O. Box. 9102, 6700 HC Wageningen, the Netherlands; E. T. M. Meekes and H. A. S. Teunissen, Naktuinbouw, P.O. Box. \\ 40, 2370 AA Roelofarendsveen, the Netherlands; and J. Van Vaerenbergh, Institute for Agricultural, Fisheries and Food Research (ILVO),
} Plant Health Department B-9820 Merelbeke, Belgium

\begin{abstract}
During the last two years, greenhouse cultivation of rose (Rosa spp.) in the Netherlands has been challenged by an uncommon bacterial disease. Affected plants suffered from chlorosis, stunting, wilting, and necrosis. The bacterial isolates obtained from the different Rosa spp. cultivars were all identified as phylotype I, sequevar 33 from the 'Ralstonia solanacearum species complex' (RSSC), actually reclassified as Ralstonia pseudosolanacearum. The work in this paper considers the genetic diversity and the phylogenetic position of 129 R. pseudosolanacearum isolates from the outbreak. This was assessed by AFLP based on four different primer combinations and MLP using partial sequences of the $e g l, m u t \mathrm{~S}$, and $f l i C$ genes. The AFLP revealed identical profiles for all the isolates, irrespective of their association with Rosa sp. propagating material, Rosa spp. plants for cut flowers, or water used in the different greenhouse cultivations. These AFLP profiles were unique and diverged

from profiles of all other reference isolates in the RSSC included. Furthermore, MLP on $e g l, f l i \mathrm{C}$, and $m u t \mathrm{~S}$ gene sequences clearly demonstrated that all $R$. pseudosolanacearum isolates clustered in phylotype $\mathrm{I}$, as a distinct monophyletic group. Interestingly, this monophyletic group also included phylotype I strain Rs-09-161 from eggplant (Solanum melongena), isolated in 2009 in India. AFLP and MLP were both efficient in revealing the genetic divergence from the RSSC isolates included. The phylogenetic tree constructed from the AFLP profiles was, in general, in agreement with the one obtained from MLP. Both phylogenetic trees displayed a similar clustering, supported by high posterior probabilities. Both methodologies clearly demonstrated that the $R$. pseudosolanacearum isolates from Rosa spp. grouped in a monophyletic group inside phylotype I, with a particular correspondence to a strain present in India, as revealed in MLP.
\end{abstract}

Ralstonia solanacearum (Smith) Yabuuchi et al. is a soilborne plant pathogenic bacterium that causes wilting, stunting, and yellowing of the foliage on more than 450 plant species and 50 botanical families. The pathogen occurs worldwide under various climatic conditions (Elphinstone 2005). It is also detected in waterways in the EU (Caruso et al. 2005; Elphinstone et al. 1998; Janse 1996; Wenneker et al. 1999), in Egypt (Tomlinson et al. 2011), and on Martinique (Wicker et al. 2007). Major economic losses caused by bacterial wilt epidemics have been produced in crop fields of tomato, potato, tobacco, banana, groundnut, and ginger. The pathogen also affects ornamental plants such as Pelargonium and Anthurium (Dookun et al. 2001; Elphinstone 2005; Hayward 1991; Poussier et al. 2000; Yik et al. 1994). Over the past decade, new plant species have been reported as hosts, particularly in South America and Asia (Chandrashekara and Prasannakumar 2010; Denny 2006; Jiang et al. 2016; Lin et al. 2014; Prieto Romo et al. 2012). Although virulence genes of $R$. solanacearum evolve and adapt at a much faster rate than its genome as a whole (Genin and Boucher 2002; Remenant et al. 2010), differential gene expression of virulence-related genes, associated with host range variation has been recently demonstrated for Moko and NPB strains (Ailloud et al. 2016).

Phenotypic properties of $R$. solanacearum attributed in the past in order to assess and categorize its heterogeneity were used to divide the species into races (Buddenhagen et al. 1962) related to virulence

${ }^{\dagger}$ Corresponding author: Maria Bergsma-Vlami; E-mail: m.vlami@nvwa.nl

Funding: This study was financially supported by research grant OS 2016339 project for $R$. solanacearum species complex of the Ministry of Economic Affairs in the Netherlands. The authors declare no conflict of interest.

*The $\boldsymbol{e}$-Xtra logo stands for "electronic extra" and indicates that one supplementary table is published online.

Accepted for publication 25 April 2018.

() 2018 The American Phytopathological Society abilities in different plant species, and into biovars related to the ability to metabolize three sugar alcohols and three disaccharides (Hayward 1964). Although widely used in the past, this division is currently considered to be of very limited value as it lacks the solid discriminatory ability that is directly associated with the broad genetic background of the pathogen (Genin and Denny 2012).

A more genetically meaningful analysis during the past decade has revealed extensive genetic diversity in $R$. solanacearum from different geographic and biological origin. This actually justified the designation of the 'Ralstonia solanacearum species complex' or RSSC, as proposed a decade ago (Fegan and Prior 2005). The RSSC was genetically differentiated into four major phylogenetic lineages or monophyletic clusters known as phylotypes. The phylotype designation is related to geographical origin of evolution: phylotype I strains have their origin in Asia, phylotype II in the Americas, phylotype III in Africa, and phylotype IV in the Indonesian archipelago, the latter phylotype including both $R$. syzygii and the banana blood disease bacterium (BDB) (Fegan and Prior 2005). A recent polyphasic taxonomic study (Safni et al. 2014) on multiple strains of the RSSC, covering a wide range of geographical and biological origins in the four phylotypes, divided the RSSC into three bacterial species. According to this study, the $R$. solanacearum is restricted to phylotype II, whereas strains of phylotype IV and BDB strains are reclassified inside the species $R$. syzygii, as subsp. syzygii and subsp. celebesensis, respectively. Phylotypes I and III form a new single bacterial species designated as $R$. pseudosolanacearum. This division has been in the meantime supported by the outcome of proteomic and genomic data (Prior et al. 2016).

Recently, we reported the finding of $R$. pseudosolanacearum ( $R$. solanacearum phylotype I) causing an unusual symptomatology and disease in Rosa spp. in greenhouse cultivation in the Netherlands (Tjou-Tam-Sin et al. 2017b). This included both Rosa spp. propagation material, as well as Rosa spp. plants for production of cut flowers. This was, worldwide, the first report of $R$. pseudosolanacearum being isolated and diagnosed from Rosa spp. The analysis of the genetic diversity of a newly introduced pathogen represents a key factor for risk assessment. This paper reports on the phylogenetic assignment among $129 R$. pseudosolanacearum isolates from the outbreak 
on Rosa spp. in the greenhouse cultivation in the Netherlands and their phylogeny in the RSSC. The analysis was done with two different molecular methodologies, namely amplified fragment length polymorphism (AFLP) and multi locus phylogeny (MLP) using three individual genes: the endoglucanase gene $e g l$, the DNA repair gene $m u t \mathrm{~S}$, and the flagellin gene $f l i \mathrm{C}$.

\section{Materials and Methods}

Bacterial isolates and culture conditions. $R$. pseudosolanacearum was isolated in 2015 and 2016 from Rosa spp. plants exhibiting the disease symptoms as previously described (Tjou-Tam-Sin et al. 2017 b). However, in some cases, $R$. pseudosolanacearum was isolated from Rosa spp. plants that exhibited no symptoms at all (latent infections). Multiple cultivars of Rosa spp. were found to be infected with $R$. pseudosolanacearum in both propagation and production companies and isolations were successful in all cases. Additionally, $R$. pseudosolanacearum was isolated from water samples from the recirculating irrigation system in the greenhouse compartments where Rosa spp. plants were cultivated. These isolates are also included in the current study (Supplementary Table S1). Isolation from plant and water samples, as well as identification and virulence testing of these $R$. pseudosolanacearum isolates, was performed as described below. In addition, a number of well characterized $R$. pseudosolanacearum and $R$. solanacearum isolates collected over a 15 -year period from various solanaceous plants, ornamental plants, and natural waterways in the Netherlands were included, along with reference isolates of worldwide origin. In total, the presented research involves 249 isolates belonging to the RSSC. A selection of 93 out of the 249 isolates, covering the currently known genetic diversity inside the RSSC (phylotypes I, II, III, and IV) and including representative $R$. pseudosolanacearum isolates from Rosa spp., was used for phylogenetic analysis. Single colony cultures were produced on nutrient agar (NA) from the isolation plates after incubation at $28^{\circ} \mathrm{C}$ for 2 to 3 days. The majority of the $R$. pseudosolanacearum isolates was routinely maintained in sterile water at room temperature by transferring one to two bacterial colonies in $3 \mathrm{ml}$ sterile water. Additionally, a selection of the $R$. pseudosolanacearum isolates was freeze-dried for prolonged preservation.

Isolation of $\boldsymbol{R}$. pseudosolanacearum from infected Rosa spp. plants and from water samples. Sampling and isolation of $R$. pseudosolanacearum from infected Rosa spp. plants and from water samples was primarily performed according the European Union protocol as described in the annexes to Commission Directive 2006/63/EC (EU 2006). Plants originated from a remarkably broad range of rose cultivars, acquired from 15 companies, including both nurseries and production of cut flowers. Both symptomatic and asymptomatic plants have been sampled and included in the analysis. Briefly, after surface sterilization, small pieces of vascular tissue $(\mathrm{ca} 0.5 \mathrm{~cm})$ originating from the basal part of the stem of symptomatic Rosa spp. plants were excised, transferred to sterile $0.01 \mathrm{M}$ phosphate buffer (PB), and allowed to soak for $30 \mathrm{~min}$ at room temperature (RT). Many symptomatic plants showed creamy brown discoloration of the vascular tissues (Fig. 1). Isolation from symptomless plants was performed by crushing stem parts (ca. $1.5 \mathrm{~cm}$ ) and transferring them to sterile $0.05 \mathrm{M}$ phosphate buffer (PB) under agitation, $100 \mathrm{rpm}$ for $30 \mathrm{~min}$ at RT. An additional centrifugation step for $15 \mathrm{~min}$ at $7,000 \times g$ was performed only for asymptomatic plant samples and the pellet was resuspended in $1.5 \mathrm{ml} \mathrm{PB} 0.01 \mathrm{M}$. A $50-\mu \mathrm{l}$ aliquot of plant extract per sample was streaked by dilution plating on plates of semiselective SMSA medium (Engelbrecht 1994 as modified by Elphinstone et al. 1996), which were then incubated for up to 6 days at $28^{\circ} \mathrm{C}$. For isolations of $R$. pseudosolanacearum from symptomatic plant samples, yeast peptone glucose agar (YPGA) medium was additionally included for dilution plating of a $20-\mu 1$ aliquot of plant extract per sample. At each rose cultivation location, water samples were taken at appropriate sites from the recirculating irrigation system in the greenhouse compartments where Rosa spp. plants were cultivated. Each water sample consisted of $2 \times 45 \mathrm{ml}$ of recirculation water. Water samples were analyzed for the presence of $R$. pseudosolanacearum by dilution plating of a $100-\mu 1$ aliquot on SMSA medium, after centrifugation. In all cases, $10 \times, 100 \times$, and
$1,000 \times$ dilutions were included for the isolations. Bacterial colonies exhibiting the morphology of the RSSC were purified after an incubation on NA for 2 to 3 days at $28^{\circ} \mathrm{C}$.

Identification of $\boldsymbol{R}$. pseudosolanacearum isolates. Typical bacterial colonies acquired from both plant and water samples were resuspended in $100 \mu \mathrm{lDNase}$ and RNase free water and heated at $95^{\circ} \mathrm{C}$ for $15 \mathrm{~min}$. After brief centrifugation to sediment cell debris, $2 \mu \mathrm{l}$ of the supernatant was used for an initial identification to RSSC by TaqMan real-time PCR using the primer combination RS-I-F/ RS-II-R (Weller et al. 2000) and the FAM/MGB labeled RSP 55T probe (Vreeburg et al. 2016). Additionally, immunofluorescence (IF) microscopy using the commercial polyclonal antiserum 073566 - C.4732 / BE021102 (LOEWE Biochemica $\mathrm{GmbH}$, Germany) was applied for identification (EU 2006), using a bacterial suspension of approximately $10^{6}$ cells $\mathrm{ml}^{-1}$. Subsequently, fatty acid analysis (FAA) was applied on a representative number of $R$. pseudosolanacearum isolates from Rosa spp. For the FAA, bacteria were grown aerobically on trypticase soy broth agar (TSBA) (Oxoid) for $48 \mathrm{~h}$ at $28^{\circ} \mathrm{C}$ before generating their fatty acid profiles (Stead et al. 1992). Fatty acid methyl ester (FAME) extraction and purification were performed as described by Stead et al. (1992) and the individual FAMEs were quantified and identified by using the Microbial Identification System software package (Microbial ID, Inc., Newark, DE).

At the proteomic level, identification of $R$. pseudosolanacearum isolates from Rosa spp. was performed using matrix-assisted laser desorption ionization time-of-flight mass spectrometry (MALDITOF MS). Bacterial isolates were grown on NA for $48 \mathrm{~h}$ at $28^{\circ} \mathrm{C}$. Database entries (MSPs) specific for the RSSC were created prior to the routine identification of $R$. pseudosolanacearum isolates from Rosa spp. and associated water samples. For whole-cell protein extraction required for the creation of the MSPs, bacterial cells were collected in $300 \mu \mathrm{l}$ water (Chromasolv LC-MS; Sigma-Aldrich, U.S.A.) and vortexed until a homogeneous suspension was reached. A volume of $900 \mu \mathrm{l}$ of absolute ethanol (100\%) (Merck) was added and the suspension was centrifuged at $20,000 \times \mathrm{g}$ for $2 \mathrm{~min}$ at RT. The acquired pellet was resuspended in equal volumes of formic acid $(70 \%)$ (Sigma-Aldrich) and acetonitrile (100\%) (Sigma-Aldrich) and the suspension was centrifuged at $20,000 \times g$ for $2 \mathrm{~min}$ at RT. One microliter of the bacterial extracts (supernatants) and $1 \mu l$ of a saturated solution of $\alpha$-cyano-4-hydroxycinnamic acid (HCCA) (Bruker Daltonics, Germany), which was used as a matrix, were deposited into a stainless plate and dried at RT prior to analysis. Eight replications per individual bacterial extract were included for the creation of the MSPs. The mass spectra profiles were obtained using a bench-top Microflex MALDI-TOF (Bruker Daltonics), including the Flex Control and Flex Analysis v3.3 software. All spectra were obtained in linear positive-ion mode with an $\mathrm{m} / \mathrm{z}$ range of 2,000 to 20,000 Da.

For the routine identification of $R$. pseudosolanacearum isolates from Rosa spp. and associated water samples, all individual isolates were included in duplicate by directly depositing harvested 2-day-old bacterial cells from NA plates onto a stainless plate. All the spectra were calibrated using a bacterial test standard preparation (BTS) of Escherichia coli DH5 $\alpha$ (Bruker Daltonics). All bacterial spectra were analyzed using Flex Analysis software (Bruker Daltonics) to generate peak lists for each isolate, and only peaks with a relative intensity greater than 2,000 Da were considered for cluster analysis.

Biovar determination of $\boldsymbol{R}$. pseudosolanacearum isolates. Isolates were assigned to a specific biovar, based on their ability to utilize or oxidize three disaccharides (sucrose, lactose, and maltose) and three sugar alcohols (mannitol, sorbitol, and dulcitol) as previously described (Hayward 1964; Hayward et al. 1990). Media were stab inoculated with pure cultures of the $R$. pseudosolanacearum isolates and incubated at $28^{\circ} \mathrm{C}$ up to 14 days.

Hypersensitivity reaction, pathogenicity, and race determination of $R$. pseudosolanacearum isolates. The ability of the $R$. pseudosolanacearum isolates to induce a hypersensitivity reaction (HR) was tested in tobacco leaves (Nicotiana tabacum L. cv. White Burley), as described by Lozano and Sequeira (1970). Fully expanded tobacco leaves (30 to 45 days from date of transplanting) were used for infiltrating a bacterial suspension of $10^{8}$ to $10^{9} \mathrm{cfu} \mathrm{ml}^{-1}$ into the intercellular 
spaces with a hypodermic syringe. Leaves were infiltrated with sterile water as negative control. Tobacco plants were maintained at $22^{\circ} \mathrm{C}$ and leaf reactions were recorded at 24 and $48 \mathrm{~h}$ after infiltration.

Virulence of $R$. pseudosolanacearum isolates was verified in planta, followed by reisolation on SMSA medium and identification in TaqMan real-time PCR (Weller et al. 2000). The pathogenicity tests were done in tomato (Solanum lycopersicum cv. Moneymaker), tobacco, and Rosa sp. (cvs. Red Naomi and Armando) plants. For tomato and tobacco, 10 seedlings were inoculated per isolate at the third true leaf stage by injecting a cell suspension of approximately $10^{7} \mathrm{cfu} \mathrm{ml}^{-1}$ in $0.01 \mathrm{M} \mathrm{PB}$ at two sites of the stem: at the lower part, just above the soil level, and a few centimeters higher. Specifically for the rose cultivars, three rooted cuttings per isolate were stem inoculated with $R$. pseudosolanacearum isolates PD 7123 and PD 7216 (both isolated from Rosa spp.) after wounding at the base of their shoot, just above the soil level, and a few centimeters higher, as previously described (Tjou-Tam-Sin et al. 2017a). Rose plants were incubated for 6 weeks at $24 / 20^{\circ} \mathrm{C}$ day/night temperature and at relative humidity $>80 \%$ with appropriate watering to avoid drought stress. The $R$. pseudosolanacearum isolates PD 1456 (phylotype I, race 1), PD 4500 (race 1), and the $R$. solanacearum PD 2762 (phylotype II, race 3) (equivalent to NCPPB $4156=$ CFBP 3857) were included as positive controls in all assays. Plants were regularly observed for symptom development such as wilting, chlorosis, plant death, and necrosis. Race determination (Buddenhagen et al. 1962) was based on the result of the test on tomato and tobacco plants and on the ability to induce an HR in tobacco leaves (Lozano and Sequeira 1970).
Phylotype determination of $\boldsymbol{R}$. pseudosolanacearum isolates. The phylotype multiplex PCR analysis (Fegan and Prior 2005) was done on bacterial colonies from pure cultures on NA, suspended in $200 \mu \mathrm{l}$ DNase and RNase free water and heated at $95^{\circ} \mathrm{C}$ for $15 \mathrm{~min}$. After brief centrifugation to sediment cell debris, $2.5 \mu 1$ of the supernatant was used in $25 \mu \mathrm{l}$ PCR reaction volumes containing Fast Start PCR Master (Roche Life Science) and $0.4 \mu \mathrm{M}$ of each primer. PCR was performed in a GeneAmp PCR system 9700 (Applied Biosystems) using the following cycle: initial denaturation at $96^{\circ} \mathrm{C}$ for $5 \mathrm{~min}$ followed by 30 cycles of $94^{\circ} \mathrm{C}$ for $30 \mathrm{~s}, 59^{\circ} \mathrm{C}$ for $30 \mathrm{~s}$, and $72^{\circ} \mathrm{C}$ for $30 \mathrm{~s}$, with a final extension of $5 \mathrm{~min}$ at $72^{\circ} \mathrm{C}$. PCR amplicons were resolved on $2 \%$ agarose gels in Tris-acetate-EDTA (TAE) buffer containing $0.5 \mu \mathrm{g} \mathrm{ml}^{-1}$ of ethidium bromide and revealed on a LKB 2011 Macro Vue Transilluminator (Pharmacia LKB Biotechnology). Amplicon sizes were estimated by comparison with a 100-bp DNA ladder (Invitrogen, Life Technologies) and to the amplicons produced by a reference strain of each phylotype, GMI1000 (phylotype I, 144 bp), LMG 9675 (phylotype II, 372 bp), CFBP 3059 (phylotype III, 91 bp), and LMG 27703 (phylotype IV, 213 bp).

AFLP analysis. An overnight fresh culture (liquid yeast peptone glucose medium; YPG) grown from a single $R$. pseudosolanacearum colony was used for DNA extraction. Cells were pelleted in a $2 \mathrm{ml}$ safe-lock Eppendorf tube ( $\max 2 \times 10^{9}$ cells) by centrifugation $(5,000 \times g$ for $10 \mathrm{~min})$ and washed with $1 \mathrm{ml}$ demineralized $\mathrm{H}_{2} \mathrm{O}$. Pelleted cells were stored at $-20^{\circ} \mathrm{C}$ until further use. DNA was extracted using the QIAGEN DNeasy Blood and Tissue kit according to the manufactures instructions for Gram-negative bacteria. DNA quality
A

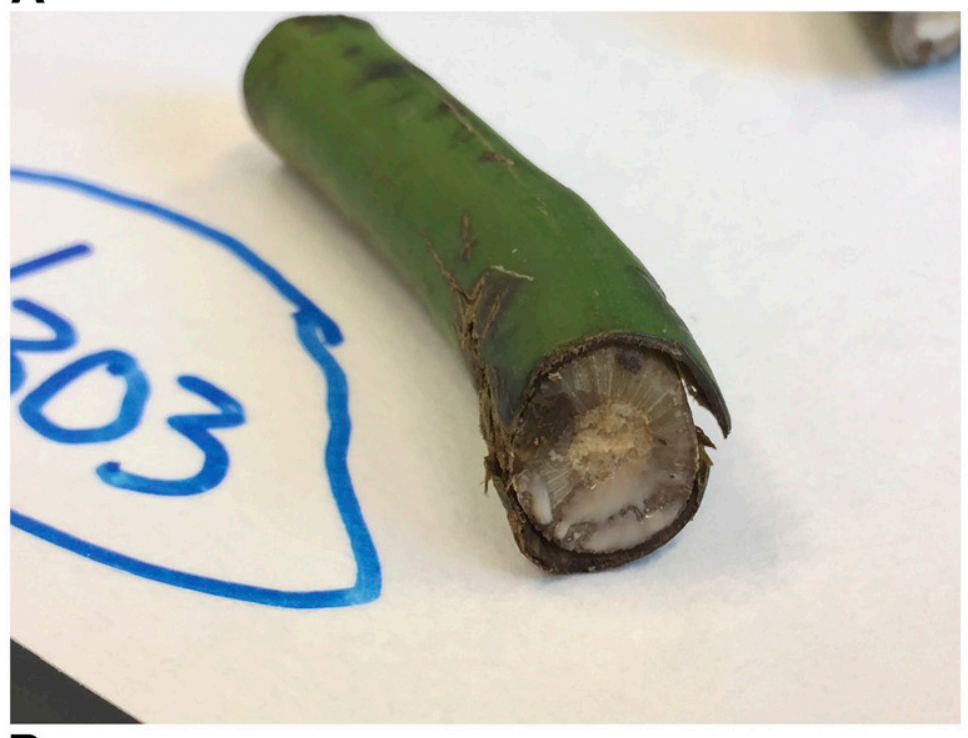

B

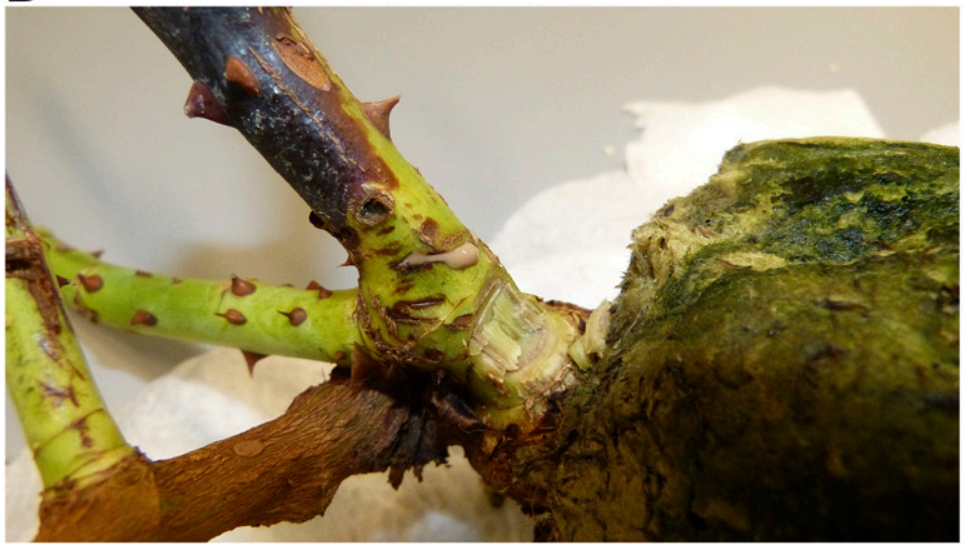

C

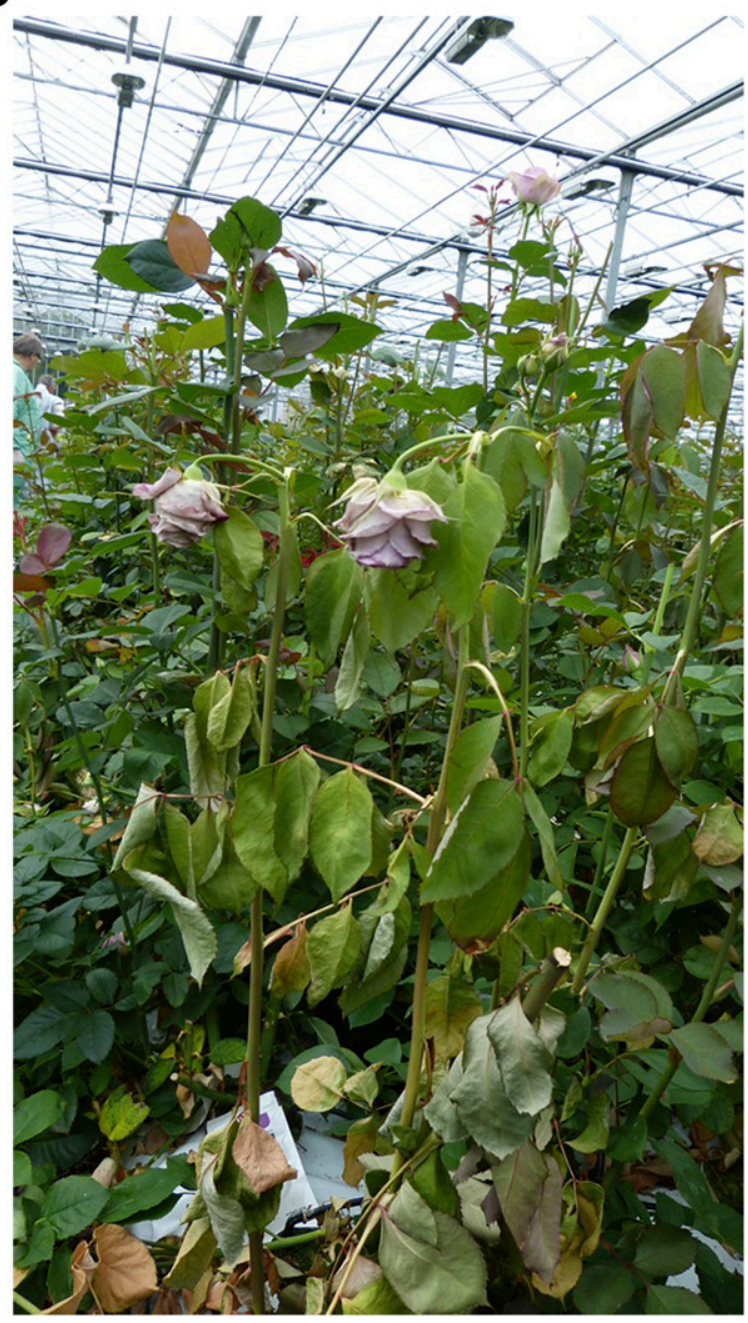

Fig. 1. A, black necrosis of pruned branches and purulent discharge of creamy white slime on cut wounds in the stem; $\mathbf{B}$, necrosis of the stems and their intense brown discoloration at their basal part, just above the ground; and $\mathbf{C}$, initial wilting of young shoots and flower stalks. 
was checked on a $1 \%$ agarose gel and DNA quantity was determined. Enzyme combination HpyCH4IV-MseI was used to fragmentize $200 \mathrm{ng}$ genomic DNA of each isolate. Subsequently, HpyCH4IVMseI-adapters were ligated according to the standard protocol based on Vos et al. (1995). The selective amplification was performed using a universal fluorescently labeled HpyCH4IV primer in combination with four non-labeled MseI primers, each with one selective base, either A (M01), T (M04), C (M02), or G (M03). After amplification, a standard set of 11 Lambda PCR fragments of known length was added to every individual reaction for normalization. DNA fragments were separated on $6 \%$ polyacrylamide gel using a Licor 4300 machine. The resulting TIFF file was imported in BioNumerics (Applied Maths). DNA profiles were normalized and only polymorphic DNA fragments were dominantly scored for their presence or absence. Genetic similarity calculations were performed on the resulting marker scores using the Jaccard similarity coefficient. Clustering analysis was performed in BioNumerics using UPGMA parameters. As a cluster verification a bootstrap analysis $(1,000 \times)$ was performed.

MLP and sequevar determination. DNA was isolated from bacterial colonies using the PrepMan Ultra kit (Thermo Fisher Scientific, Bleiswijk, The Netherlands) in a final volume of $100 \mu 1$ DNase and RNase free water. The purified DNA was used to PCR amplify the $e g l$ and $m u t$ S loci according to Wicker et al. (2007) using primer pairs Endo-F/Endo-R and mutS-RsF.1570/mutS-RsR1926, respectively. The fliC locus was PCR amplified using primer pair Rsol_fli_for/ Rsol_fli_rev according to Schönfeld et al. (2003). PCR amplicons were purified using the DNA Clean \& Concentrator-25 kit (Zymo Research, Freiburg, Germany) and sequenced using BigDye v3.1 chemistry (Thermo Fisher Scientific) and sample specific primers. The sequencing runs were performed on a $3730 \mathrm{XL}$ or 3730 Genetic analyzer (Thermo Fisher Scientific). Consensus sequences were generated from both trace files in Geneious R10 (Biomatters, Auckland, New Zealand). Amplification primers were trimmed from the consensus sequence, and when needed, additional trimming was performed to obtain high quality (PHRED > 30) consensus sequence. Sequences of each loci were aligned using MAFFT (Katoh et al. 2002) in Geneious R10 (Biomatters). Alignments were concatenated using SequenceMatrix 1.8 (Vaidya et al. 2011) with external gaps and missing sequences recoded as question marks. Phylogenetic analyses of the (concatenated) sequence alignment(s) was performed with Bayesian inference (BI) using MrBayes 3.2.1 (Huelsenbeck and Ronquist 2001). The optimal model for nucleotide substitution per locus was obtained with JModelTest 2.1.10 (Darriba et al. 2012) with AIC, AICc, BIC, and DT using default settings. Analysis of Bayesian inference was performed with a random starting tree and four Monte Carlo Markov chains for $1.1 \times 10^{6}$ generations under the HKI model with I distribution for the $e g l$ and $f l i C$ loci and G distribution for the mutS locus. Trees were sampled every 200 generations. The first $1 \times$ $10^{5}$ generations were discarded as burn-in, and the remaining trees combined to generate a $50 \%$ majority rule consensus tree that represented posterior probabilities. Partial sequences of the $\mathrm{egl}$ gene (accession nos. MF141027, MF141028, MF141029), the fliC gene (MF141024, MF141025, MF141026), and the mutS gene (MF141030 and MF141031), acquired from isolates PD 7123, PD 7195, and PD 7216, respectively, were deposited in GenBank (https://www.ncbi. nlm.nih.gov/genbank). The sequevar determination of the R. pseudosolanacearum isolates from the outbreak on Rosa spp. was based on the partial sequences of the $e g l$ gene as previously described (Fegan and Prior 2005).

\section{Results}

Symptomatology of $R$. pseudosolanacearum assessed on naturally-infected Rosa spp. During the onset of the outbreak in the early autumn of 2015, an unusual disorder was observed in various rose cultivars. Initially, wilting of young shoots and flower stalks was observed, followed by yellowing of leaves, stunting, dieback with black necrosis of pruned branches, and in some cases, purulent discharge of creamy white oozes (Fig. 1) on cut wounds in the stem. Typical symptoms from heavy infections included the necrosis of the stems and their intense brown discoloration at their basal part, just above the ground (Fig. 1). A bacterial wilt infection caused by R. pseudosolanacearum was confirmed and disease incidence often reached the $50 \%$ of the cultivated plants. Attempts to trace back the possible pathway of $R$. pseudosolanacearum infection primarily resulted in additional findings in more than 40 rose cultivars, including both propagation plant material, as well as plants for production of cut flowers.

Isolation of $R$. pseudosolanacearum from infected Rosa spp. plants and associated water. $R$. pseudosolanacearum is relatively easy to isolate from infected plant material and water samples on semiselective medium SMSA or, in cases of symptomatic plant material, on YPGA medium. $R$. pseudosolanacearum was isolated from: 1) plants exhibiting typical symptoms, 2) plants without showing any symptomatology at all (latent material), and 3) water samples obtained from the recirculating irrigation system in the greenhouse compartments where plants were cultivated. Individual colonies exhibiting typical morphology on SMSA or YPGA medium were selected for identification. On SMSA, typical bacterial colonies appeared to be fluidal, turbid purplish, white with pink centers, with irregular shape after 2 to 6 days incubation at $28^{\circ} \mathrm{C}$. In a total number of 115 plant samples, $R$. pseudosolanacearum was detected and isolated on SMSA and/or YPGA medium. These 115 Rosa spp. samples originated from a remarkably broad range of rose cultivars. Samples were analyzed from at least 40 rose cultivars from 15 companies, including both nurseries and production of cut flowers. From the 115 $R$. pseudosolanacearum isolates acquired in total, 15 isolates originated from symptomatic plants and 100 isolates from plants exhibiting no symptomatology. From the water samples, 14 were found to be positive for $R$. pseudosolanacearum and isolates were obtained and included in this study as well. Relatively high densities of $R$. pseudosolanacearum were detected in both symptomatic and asymptomatic plant material. $R$. pseudosolanacearum presented densities above $2 \times 10^{7} \mathrm{cfu} \mathrm{ml}^{-1}$ on SMSA medium and above $5 \times 10^{7}$ cfu $\mathrm{ml}^{-1}$ on YPGA medium in symptomatic plants. Asymptomatic Rosa sp. plants commonly contained similar inoculum densities, generally above $2 \times 10^{7} \mathrm{cfu} \mathrm{ml}^{-1}$ on SMSA medium, and only in a restricted number of asymptomatic plants were inoculum densities of $R$. pseudosolanacearum lower, i.e., between $2 \times 10^{3}$ and $1 \times 10^{6}$ cfu $\mathrm{ml}^{-1}$. In the associated water samples, densities of $R$. pseudosolanacearum were between $2 \times 10^{4}$ and $3 \times 10^{6} \mathrm{cfu} \mathrm{ml}^{-1}$.

Identification of $\boldsymbol{R}$. pseudosolanacearum isolates. The 129 $R$. pseudosolanacearum isolates originating from the above described samples were identified in the RSSC by application of the TaqMan real-time PCR described by Weller et al. (2000). Additionally, IF microscopy confirmed the positive real-time PCR results. Using FAME/MIDI analysis, isolates were accurately identified as belonging to RSSC and gave in the FAA a similarity index that varied between 0.69 and 0.80 based on the PD 1.90 method. However, FAME/ MIDI analysis could not differentiate isolates according to race, biovar, geographic origin, or host plant. At the proteomic level using MALDI-TOF MS, the identity of $R$. pseudosolanacearum isolates was confirmed. Scores between 2,342 and 2,543 were obtained when the commercial library of Bruker Daltonics was amended by the MSPs related to RSSC, indicating that the MSPs created before the routine identification of the $R$. pseudosolanacearum isolates are very specific for the RSSC and can be successfully used in combination with the commercial library of Bruker Daltonics.

Biovar, HR, pathogenicity, and race determination of $\boldsymbol{R}$. pseudosolanacearum isolates. The $R$. pseudosolanacearum isolates produced positive results in the disaccharides (sucrose, lactose, and maltose) and sugar alcohols (mannitol, sorbitol, and dulcitol). These biochemical tests attributed the $R$. pseudosolanacearum isolates to biovar 3. Necrosis (HR+) was assessed in the tobacco leaves after 24 to $48 \mathrm{~h}$. Stem inoculations in tomato, in tobacco, and in rooted rose cuttings (cvs. Red Naomi and Armando) resulted in clear wilting symptoms, often including necrosis of plant tissue. $R$. pseudosolanacearum was reisolated on SMSA medium and identified by the TaqMan real-time PCR of Weller et al. (2000). Based on the virulence of the R. pseudosolanacearum isolates in tomato and in tobacco plants, as well as their HR in tobacco leaves, these isolates fit to race 1 . 
Phylotype determination of $\boldsymbol{R}$. pseudosolanacearum isolates. The $R$. pseudosolanacearum isolates from naturally infected Rosa spp. plants and associated water samples produced the 144-bp amplicon in the phylotype multiplex PCR which assigns them to phylotype I.

AFLP analysis. The total number of markers designated as bands on the gel for the 243 profiled isolates was approximately 560 . Of these, the majority were polymorphic, indicating the broad genetic diversity inside the RSSC. We used 303 polymorphic markers that proved to be reproducible and reliable among all isolates. The number of polymorphic markers used was highest in the primer combination H00-M02 (98 markers). The primer combination H00-M01 resulted in 72 polymorphic markers. Primer combinations H00-M03 and H00-M04 contributed to the number of polymorphic markers in this study with 53 and 80 polymorphic markers, respectively. The overall observed genetic diversity in the RSSC was very broad as indicated by the low similarity values between diverse clusters representing the phylotype I, II, III, and IV (Jaccard similarities below 0.20). The three recently described species inside the RSSC are clearly separated in distinct clades (Fig. 2). Despite the broad genetic diversity found in the RSSC, no genetic diversity among the $R$. pseudosolanacearum isolates from the rose plants and the associated water samples was observed. AFLP analysis showed identical profiles for these isolates that clustered inside the phylotype I group. Within the phylotype I clade, the closest genetic related subcluster contained isolates from a diversity of host plants, e.g., anthurium, tomato, dahlia, vicia, and strelitzia, all originating from Africa. However, the genetic similarities were lower than 0.50 , indicating that the Rosa sp. cluster was clearly distinct. Additionally, all $R$. pseudosolanacearum isolates originating from the naturally infected Rosa spp. plants and the associated water samples clustered separately to the Curcuma phylotype I cluster previously found in the Netherlands, as well as to diverse phylotype I isolates originating from Central and South America, Southeast Asia, and Oceania (Fig. 2).

MLP and sequevar determination. The majority of isolates and strains (179 of the 249 isolates, including all those from rose cultivation and associated irrigation water and others from diverse host plants) were classified in phylotype I of the RSSC in the MLP analysis of individual and concatenated $e g l, f l i \mathrm{C}$, and $m u t \mathrm{~S}$ gene sequences. Therefore, these isolates were identified as $R$. pseudosolanacearum.

The $R$. pseudosolanacearum isolates from the rose cultivation and the associated irrigation water (Fig. 3) formed a distinct monophyletic clade in phylotype I. The $100 \%$ sequence homology implies that all these isolates are descendants of a common ancestor and that they might have recently been introduced. Interestingly, the clade containing the isolates from rose cultivation also included strain Rs-09-161 from eggplant (Solanum melongena) isolated in 2009 in India (Ramesh et al. 2014a, b). The egl sequences (accession nos. MF141027, MF141028, MF141029), the fliC sequences (MF141024, MF141025, MF141026), and the mutS sequences (MF141030 and MF141031) from three representative $R$. pseudosolanacearum isolates from Rosa sp. (PD 7123, PD 7195, and PD 7216) and from phylotype I isolate Rs-09-161 (HQ245025 and JHBO00000000) from India (Ramesh et al. 2014a, b) were found to be $100 \%$ identical. The phylogenetic analysis based only on the egl gene sequence confirmed these findings (Fig. 4). The clade closest to the $R$. pseudosolanacearum rose isolates is containing phylotype I isolates from Africa, such PD 5415 from anthurium, supported by a high posterior probability (0.92). Additionally, they clustered separately from the Curcuma phylotype I cluster previously found in the Netherlands, as well as to diverse phylotype I isolates originating from Central and South America, Southeast Asia, and Oceania. The tree constructed from the concatenated partial sequences of $e g l, f l i \mathrm{C}$, and $m u t \mathrm{~S}$ was in accordance with the tree constructed from the partial sequences of egl alone (Figs. 3 and 4), showing a similar clustering, supported by high posterior probabilities (1). The sequevar determination of the $R$. pseudosolanacearum isolates from Rosa spp. revealed that they belong to sequevar I-33, as their egl sequences (MF141027, MF141028, MF141029) were 99.9\% identical to egl sequences (KX242313 to KX242316) from R. pseudosolanacearum (phylotype
I) strains CFBP 8467, CFBP 8468, CFBP 8469, and CFBP 8466 causing bacterial wilt on Rodrigues Island in the Indian Ocean (Yahiaoui et al. 2016).

\section{Discussion}

The RSSC is considered as a group of genetically diverse but pathologically closely related vascular pathogens that can rapidly spread from mother plants to their progeny through vegetative propagation, i.e., in potato seed tubers, rhizomes, and cuttings (Elphinstone 2005).

Infection can remain latent when the interaction with the host is not conducive for symptom development. This is particularly risky for nurseries because latently infected plants are propagated and distributed over many growers. The plants remain asymptomatic until the interaction with the host favors symptom expression, i.e., the pathogen overpowers the plant's defense. As latently infected plants pose the greatest risk for undeliberate spread of pathogens, the ability of RSSC pathogens to occur frequently as latent infections has become a serious challenge for the floricultural industry, as already demonstrated for Pelargonium (Swanson et al. 2007), Anthurium (Dookun et al. 2001; Poussier et al. 2000; Yik et al. 1994), and other ornamental plants (Norman et al. 2009). Latent infection does not imply that low or undetectable bacterial densities are present in the plant. On the contrary, in particular for the RSSC pathogens, the bacterial population density accumulating inside the plant might be at a high level, up to $7 \times 10^{5} \mathrm{cfu} \mathrm{ml}^{-1}$ (Swanson et al. 2007). In our study, relatively high inoculum densities of $R$. pseudosolanacearum were detected in infected but asymptomatic Rosa spp. plant material, often up to $2 \times 10^{7} \mathrm{cfu} \mathrm{ml}^{-1}$. Additionally, RSSC pathogens can survive for years in waterways (Álvarez et al. 2008; van Overbeek et al. 2004), they can successfully enter and colonize the root system of a host plant and rapidly spread to the above ground part of the plant, in a systemic way through the vascular tissues, often leading to major wilt symptomatology. $R$. pseudosolanacearum inoculum densities ranging from $2 \times 10^{4}$ to $3 \times 10^{6} \mathrm{cfu} \mathrm{ml}^{-1}$ were detected in the water samples taken from the recirculating irrigation system in the greenhouse compartments where Rosa spp. plants were cultivated, underlining the high risk for pathogen dissemination through the recirculating water. Currently, many ornamentals are cultivated in Africa, Central America, and Southeast Asia, where they may encounter new pathogens or new variants of existing pathogens. The rooted cuttings harboring these pathogens are then exported to Europe. Contaminated irrigation water and infected planting material represent crucial pathways for long distance dispersal of RSSC (Elphinstone 2005). The probability of a major dispersal is substantially increased if the absence of RSSC in irrigation water and in the mother plants is not guaranteed. The notorious association of $R$. pseudosolanacearum with roses being previously unknown, propagation and cultivation in nurseries was not tested, nor surveyed for the eventual presence of this pathogen.

This paper reports on the characterization of $R$. pseudosolanacearum isolates from the Rosa spp. plants and the associated irrigation water, as well as their phylogenetic relatedness with other strains in the RSSC. Both molecular methods applied in the present study, AFLP and MLP, were valid and conclusive to classify the RSSC isolates. The clustering according to the AFLP markers was similar to the tree obtained from the three partial gene sequences of $e g l, f l i C$, and mutS in MLP. The corresponding clustering confirmed the four phylotypes with high values. Additionally, both methodologies clearly assigned the isolates from the rose cultivation to a single cluster in phylotype I. Furthermore, the concatenated partial gene sequences of $e g l, f l i \mathrm{C}$, and $m u t \mathrm{~S}$ and the partial sequences of the $e g l$ alone displayed similar classification. Phylogenetic analysis of the $e g l$ gene sequence provided a valid identification of the isolates from the rose cultivation. The $e g l$ gene is a good estimator of the natural phylogeny of the RSSC (Fegan and Prior 2005). Furthermore, MLP based on $e g l, f l i \mathrm{C}$, and $m u t \mathrm{~S}$ presented in this study was in agreement with previous work (Wicker et al. 2012; Yahiaoui et al. 2017). Because of the global trading of ornamentals, it is essential to use a tool that is able to determine the geographic or evolutionary origin of the bacterial wilt outbreaks due to RSSC. The $e g l$ gene, 
among other genes, has been used to characterize individual isolates of RSSC down to the continent or the country of origin (Villa et al. 2005). Interestingly, the clade containing the $R$. pseudosolanacearum isolates from the rose cultivation also included strain Rs-09161 isolated in 2009 from eggplant (S. melongena), showing 100\% homology on $e g l, f l i \mathrm{C}$, and $m u t \mathrm{~S}$ sequencies. Its geographical origin is the coastal region of Goa, India (Ramesh et al. 2014a, b). Whether isolate Rs-09-161 can infect Rosa spp. and result in bacterial wilt symptomatology remains undetermined. Full genome sequencing of a $R$. pseudosolanacearum isolate from Rosa sp. would allow comparison with the genome of the Indian isolate (Ramesh et al. 2014b). The associated $R$. pseudosolanacearum populations on the different

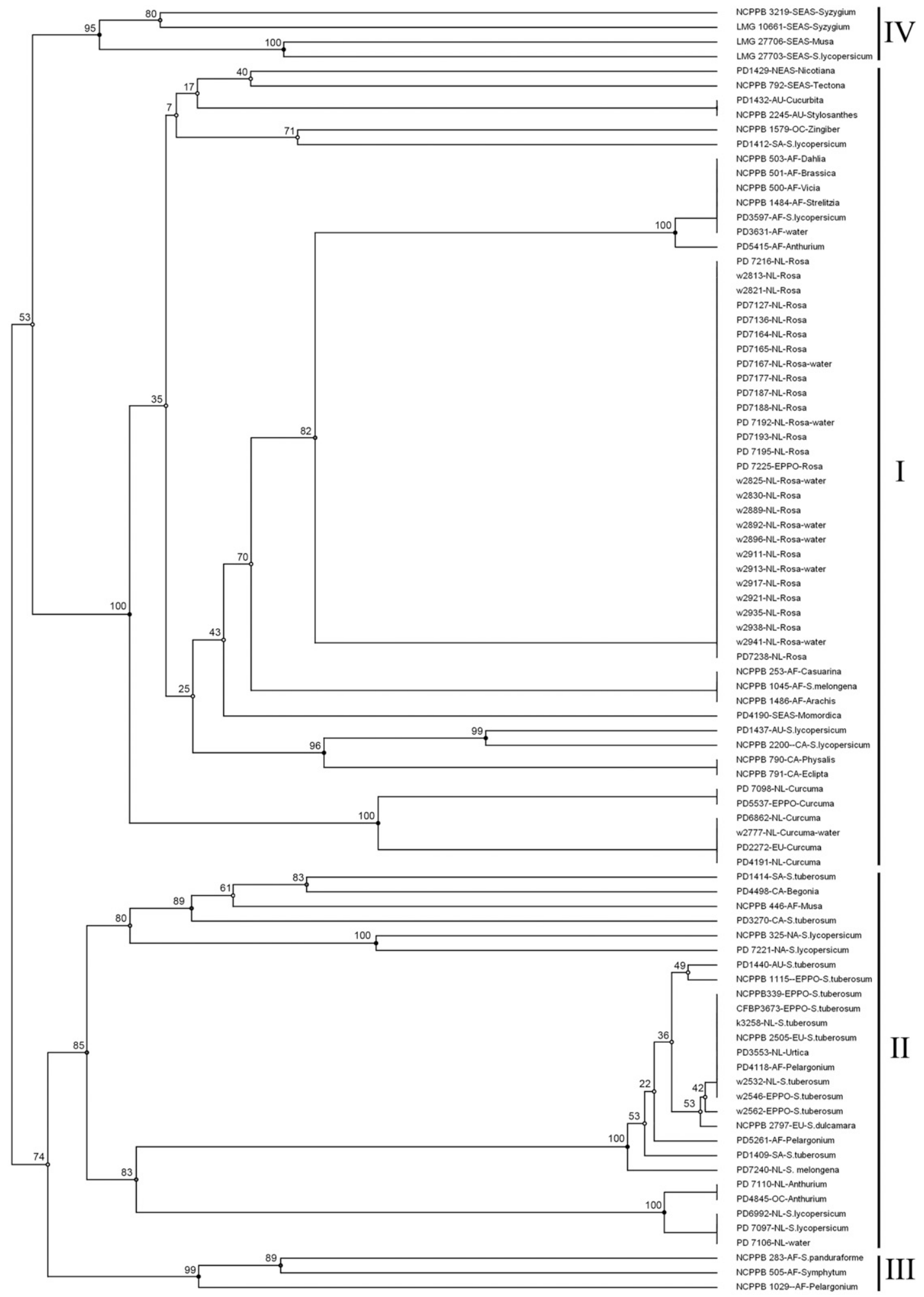

Fig. 2. Phylogenetic analysis of Ralstonia pseudosolanacearum isolates from Rosa spp. and related RSSC isolates using amplified fragment length polymorphism (AFLP) data. Analysis was based on 303 polymorphic markers. DNA profiles were normalized and were scored for their presence or absence. Genetic similarity calculations were performed on the resulting marker scores using the Jaccard similarity coefficient. Clustering analysis was performed in BioNumerics software using UPGMA algorithm. I, II, III, or IV indicates the phylotype. 
Rosa spp. cultivars were genetically homogeneous and are all assigned to phylotype I, sequevar I-33, race 1, biovar 3. R. pseudosolanacearum isolates belonging to phylotype I and sequevar I-33 have been previously found in several African countries, among them Kenya (S. lycopersicum and in water), Ghana (Anthurium andreanum), and the southwest Indian Ocean islands, including Madagascar (S. lycopersicum and Solanum tuberosum), Reunion (S. lycopersicum and Anthurium sp.), Mauritius (Strelingia reginal, Vicia faba,
Brassica oleracea, Dahlia sp., S. tuberosum, S. lycopersicum, and A. andreanum), and more recently in Rodrigues (S. lycopersicum and Capsicum frutescens) (Dookun et al. 2001; Poussier et al. 2000; Yahiaoui et al. 2016, 2017, unpublished data). Additionally, several of these $R$. pseudosolanacearum isolates have been characterized as belonging to race 1 and biovar 3 , confirming the hypothesis that several race 1 isolates can infect a broad range of plant species and that biovar 3 isolates exhibit an intermediate host range, between

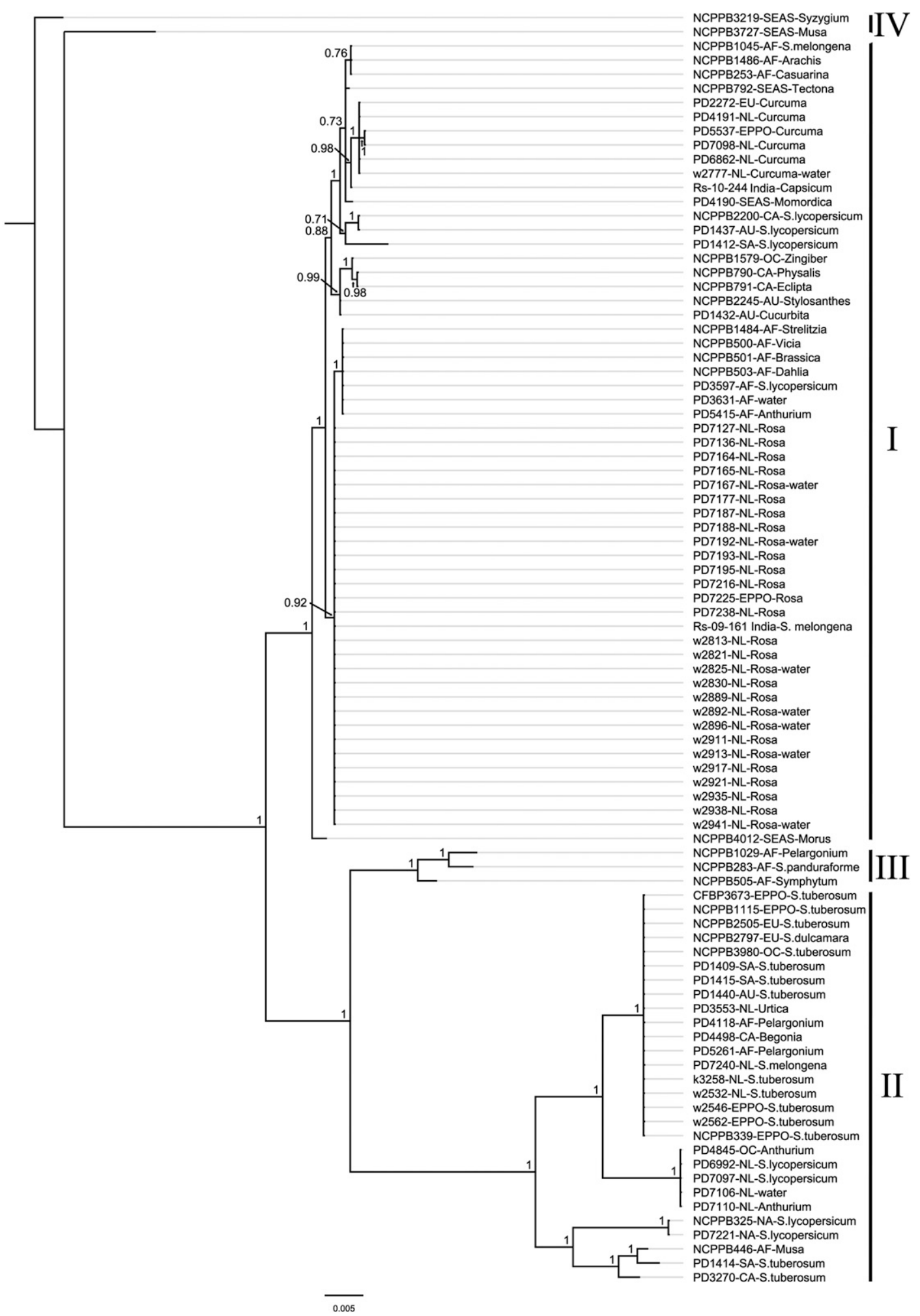

Fig. 3. Phylogenetic analysis of Ralstonia pseudosolanacearum isolates from Rosa spp. and related RSSC isolates based on Bayesian analysis of egl, mutS, and fliC concatenated sequences. Posterior probabilities are indicated at the nodes. Bar represents genetic distances in nucleotide substitutions per site. Ralstonia syzygii strain NCPPB 3219 is used as out-group. I, II, III, or IV indicates the phylotype. 
the immense host range of biovar 1 isolates and the rather restricted host range of biovar 2 isolates (Elphinstone 2005).

Global trading significantly contributes to the pathogen's rapid spread over long distances, their high recombination frequencies, and high adaptation. Recombination has recently been shown to play a major role in the evolution of $R$. solanacearum genome (Peeters et al. 2013; Wicker et al. 2012; Yahiaoui et al. 2017) that might end up in new epidemic diseases. Interestingly, phylotype I exhibited the highest recombination events among all four phylotypes and has rapidly spread over long distances, most probably as latent infections in plant material and very adaptive in tropical conditions (Wicker et al. 2012). In general, phylotype 1 strains of $R$. pseudosolanacearum are most destructive in tropical and subtropical climates; however, they can incidentally cause significant losses in ornamentals under glass.

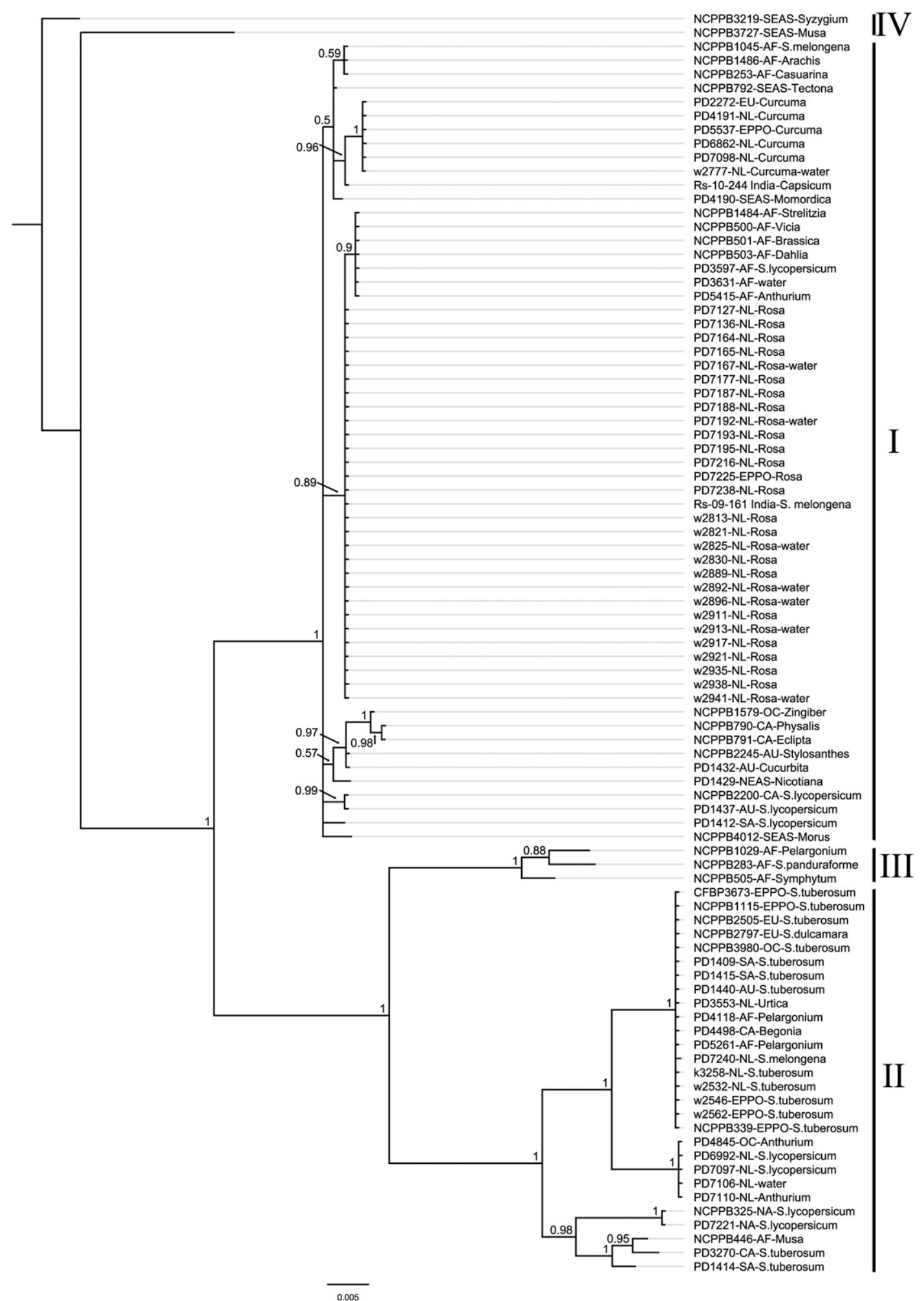

Fig. 4. Phylogenetic analysis of Ralstonia pseudosolanacearum isolates from Rosa spp. and related RSSC isolates based on Bayesian analysis of egl sequences only. Posterior probabilities are indicated at the nodes. Bar represents genetic distances in nucleotide substitutions per site. Ralstonia syzygii strain NCPPB 3219 is used as out-group. I, II, III, or IV indicates the phylotype. 


\section{Literature Cited}

Ailloud, F., Lowe, T. M., Robène, I., Cruveiller, S., Allen, C., and Prior, P. 2016. In planta comparative transcriptomics of host-adapted strains of Ralstonia solanacearum. PeerJ 4:e1549.

Álvarez, B., López, M. M., and Biosca, E. G. 2008. Survival strategies and pathogenicity of Ralstonia solanacearum phylotype II subjected to prolonged starvation in environmental water microcosms. Microbiology 154:3590-3598.

Buddenhagen, I., Sequeira, L., and Kelman, A. 1962. Designation of races in Pseudomonas solanacearum. Phytopathology 52:726.

Caruso, P., Palomo, J. L., Bertolini, E., Álvarez, B., López, M. M., and Biosca, E. G. 2005. Seasonal variation of Ralstonia solanacearum biovar 2 populations in a Spanish river: recovery of stressed cells at low temperatures. Appl. Environ. Microbiol. 71:140-148.

Chandrashekara, K. N., and Prasannakumar, M. K. 2010. New host plants for Ralstonia solanacearum from India. Plant Pathol. 59:1164.

Darriba, D., Taboada, G. L., Doallo, R., and Posada, D. 2012. JModelTest 2: more models, new heuristics and parallel computing. Nat. Methods 9:772.

Denny, T. 2006. Plant pathogenic Ralstonia species. Pages 573-644 in: Plantassociated bacteria. S. S. Gnanamanickam, ed. Springer Netherlands, Dordrecht, The Netherlands.

Dookun, A., Saumtally, S., and Seal, S. 2001. Genetic diversity in Ralstonia solanacearum strains from Mauritius using restriction fragment length polymorphisms. Phytopathol. Z. 149:51-55.

EU. 2006. EC 2006 Council directive 2006/63/EC. Official J. Eur. Commun. L206:1-106.

Elphinstone, J. G. 2005. The current bacterial wilt situation: a global overview. Pages 9-28 in: Bacterial Wilt Disease and the Ralstonia solanacearum Species Complex. C. Allen, P. Prior, and A. C. Hayward, eds. American Phytopathological Society, St. Paul, MN.

Elphinstone, J. G., Hennessy, J., Wilson, J. K., and Stead, D. 1996. Sensitivity of different methods for the detection of Ralstonia solanacearum in potato tuber extracts. EPPO Bull. 26:663-678.

Elphinstone, J. G., Stanford, H. M., and Stead, D. E. 1998. Detection of Ralstonia solanacearum in potato tubers, Solanum dulcamara, and associated irrigation water. Pages 133-139 in: Bacterial Wilt Disease: Molecular and Ecological Aspects. P. Prior, C. Allen, and J. Elphinstone, eds. Springer publishing, Berlin.

Engelbrecht, M. C. 1994. Modification of a selective medium for the isolation and quantification of Pseudomonas solanacearum. ACIAR Bacterial Newsl. 10:3-5.

Fegan, M., and Prior, P. 2005. How complex is the "Ralstonia solanacearum species complex"? Pages 449-461 in: Bacterial Wilt Disease and the Ralstonia solanacearum Species Complex. C. Allen, P. Prior, and A. C.Hayward, eds. American Phytopathological Society, St. Paul, MN.

Genin, S., and Boucher, C. 2002. Ralstonia solanacearum: Secrets of a major pathogen unveiled by analysis of its genome. Mol. Plant Pathol. 3:111-118.

Genin, S., and Denny, T. P. 2012. Pathogenomics of the Ralstonia solanacearum species complex. Annu. Rev. Phytopathol. 50:67-89.

Hayward, A. C. 1964. Characteristics of Pseudomonas solanacearum. J. Appl. Bacteriol. 27:265-277.

Hayward, A. C. 1991. Biology and epidemiology of bacterial wilt caused by Pseudomonas solanacearum. Annu. Rev. Phytopathol. 29:65-87.

Hayward, A. C., El-Nashaar, H. M., Nydegger, U., and De Lindo, L. 1990. Variation in nitrate metabolism in biovars of Pseudomonas solanacearum. J. Appl. Bacteriol. 69:269-280.

Huelsenbeck, J. P., and Ronquist, F. 2001. MRBAYES: Bayesian inference of phylogenetic trees. Bioinformatics 17:754-755.

Janse, J. D. 1996. Potato brown rot in Western Europe - history, present occurrence and some remarks on possible origin, epidemiology and control strategies. EPPO Bull. 26:679-695.

Jiang, Y., Li, B., Liu, P., Liao, F., Weng, Q., and Chen, Q. 2016. First report of bacterial wilt caused by Ralstonia solanacearum on fig trees in China. For. Pathol. 46:256-258.

Katoh, K., Misawa, K., Kuma, K., and Miyata, T. 2002. MAFFT: a novel method for rapid multiple sequence alignment based on fast Fourier transform. Nucleic Acids Res. 30:3059-3066.

Lin, C. H., Tsai, K. C., Prior, P., and Wang, J. F. 2014. Phylogenetic relationships and population structure of Ralstonia solanacearum isolated from diverse origins in Taiwan. Plant Pathol. 63:1395-1403.

Lozano, J. C., and Sequeira, L. 1970. Differentiation of races of Pseudomonas solanacearum by a leaf infiltration. Phytopathology 60:833-838.

Norman, D. J., Zapata, M., Gabriel, D. W., Duan, Y. P., Yuen, J. M. F., Mangravita-Novo, A., and Donahoo, R. S. 2009. Genetic diversity and host range variation of Ralstonia solanacearum strains entering North America. Phytopathology 99:1070-1077.

Peeters, N., Carrere, S., Anisimova, M., Plener, L., Cazale, A.-C., and Genin, S. 2013. Repertoire, unified nomenclature and evolution of the type III effector gene set in the Ralstonia solanacearum species complex. BMC Genomics 14:859.

Poussier, S., Trigalet-Demery, D., Vandewalle, P., Goffiner, B., Luisetti, J., and Trigalet, A. 2000. Genetic diversity of Ralstonia solanacearum as assessed by PCR-RFLP of the hrp region, AFLP and 16S rRNA sequence analysis, and identification of an African subdivision. Microbiology 146: 1679-1692.

Prieto Romo, J., Morales Osorio, J. G., and Salazar Yepes, M. 2012. Identification of new hosts for Ralstonia solanacearum (Smith) race 2 from Colombia. Rev. Prot. Veg. 27:151-161.

Prior, P., Ailloud, F., Dalsing, B. L., Remenant, B., Sanchez, B., and Allen, C. 2016. Genomic and proteomic evidence support the division of the plant pathogen Ralstonia solanacearum into three species. BMC Genomics 17: 90

Ramesh, R., Achari, G. A., and Gaitonde, S. 2014a. Genetic diversity of Ralstonia solanacearum infecting solanaceous vegetables from India reveals the existence of unknown or newer sequevars of Phylotype I strains. Eur. J. Plant Pathol. 140:543-562.

Ramesh, R., Gaitonde, S., Achari, G., Asolkar, T., Singh, N. P., Carrere, S., Genin, S., and Peeters, N. 2014b. Genome sequencing of Ralstonia solanacearum biovar 3, phylotype I, strains Rs-09-161 and Rs-10-244, isolated from eggplant and chili in India. Genome Announc. 2:e00323-e14.

Remenant, B., Coupat-Goutaland, B., Guidot, A., Cellier, G., Wicker, E., and Allen, C. 2010. Genomes of three tomato pathogens within the Ralstonia solanacearum species complex reveal significant evolutionary divergence. BMC Genomics 11:379.

Safni, I., Cleenwerck, I., De Vos, P., Fegan, M., Sly, L., and Kappler, U. 2014 Polyphasic taxonomic revision of the Ralstonia solanacearum species complex: proposal to emend the descriptions of Ralstonia solanacearum and Ralstonia syzygii and reclassify current $R$. syzygii strains as Ralstonia syzygii subsp. syzygii subsp. nov., $R$. solanacearum phylotype IV strains as Ralstonia syzygii subsp. indonesiensis subsp. nov., banana blood disease bacterium strains as Ralstonia syzygii subsp. celebesensis subsp. nov. and $R$. solanacearum phylotype I and III strains as Ralstonia pseudosolanacearum sp. nov. Int J. Syst. Evol. Microbiol. 64:3087-3103.

Schönfeld, J., Heuer, H., Van Elsas, J. D., and Smalla, K. 2003. Specific and sensitive detection of Ralstonia solanacearum in soil on the basis of PCR amplification of fliC fragments. Appl. Environ. Microbiol. 69: 7248-7256.

Stead, D. E., Sellwood, J. E., Wilson, J., and Viney, I. 1992. Evaluation of a commercial microbial identification system based on fatty acid profiles for rapid, accurate identification of plant pathogenic bacteria. J. Appl. Bacteriol. 72:315-321.

Swanson, J. K., Montes, L., Mejia, L., and Allen, C. 2007. Detection of latent infections of Ralstonia solanacearum race 3 biovar 2 in geranium. Plant Dis. 91:828-834.

Tjou-Tam-Sin, N. N. A., van de Bilt, J. L. J., Westenberg, M., Gorkink-Smits, P., Landman, N. M., and Bergsma-Vlami, M. 2017a. Assessing the pathogenic ability of Ralstonia pseudosolanacearum (Ralstonia solanacearum Phylotype I) from ornamental Rosa spp. plants. Front. Plant Sci. 8:1895.

Tjou-Tam-Sin, N. N. A., van de Bilt, J. L. J., Westenberg, M., Korpershoek, H. J. Vermunt, A. M. W., Meekes, E. T. M., Teunissen, H. A. S., and Van Vaerenbergh, J. 2017b. First Report of Bacterial Wilt Caused by Ralstonia solanacearum in Ornamental Rosa sp. Plant Dis. 101:378.

Tomlinson, D. L., Elphinstone, J. G., El-Fatah, H. A., Agag, S., and Kamal, M. 2011. Limited survival of Ralstonia solanacearum race 3 in bulk soils and composts from Egypt. Eur. J. Plant Pathol. 131:197-209.

Vaidya, G., Lohman, D. J., and Meier, R. 2011. SequenceMatrix: concatenation software for the fast assembly of multi-gene datasets with character set and codon information. Cladistics 27:171-180.

van Overbeek, L. S., Bergervoet, J. H. W., Jacobs, F. H. H., and van Elsas, J. D. 2004. The low-temperature-induced viable-but-nonculturable state affects the virulence of Ralstonia solanacearum biovar 2. Phytopathology 94:463-469.

Villa, J. E., Tsuchiya, K., Horita, M., Opina, N., and Hyakumachi, M. 2005. Phylogenetic relationships of Ralstonia solanacearum species complex strains from Asia and other continents based on 16S rDNA, endoglucanase, and $h r p \mathrm{~B}$ gene sequences. J. Gen. Plant Pathol. 71:39-46.

Vos, P., Hogers, R., Bleeker, M., Reijans, M., van de Lee, T., Hornes, M., Frijters, A., Pot, J., Peleman, J., and Kuiper, M. 1995. AFLP: A new technique for DNA fingerprinting. Nucleic Acids Res. 23:4407-4414.

Vreeburg, R. A. M., Bergsma-Vlami, M., Bollema, R. M., de Haan, E. G., Kooman-Gersmann, M., Smits-Mastebroek, L., Tameling, W. I. L., TjouTam-Sin, N. N. A., van de Vossenberg, B. T. L. H., and Janse, J. D. 2016. Performance of real-time PCR and immunofluorescence for the detection of Clavibacter michiganensis subsp. sepedonicus and Ralstonia solanacearum in potato tubers in routine testing. EPPO Bull. 46:112-121.

Weller, S. A., Elphinstone, J. G., Smith, N. C., Boonham, N., and Stead, D. E. 2000. Detection of Ralstonia solanacearum strains with a quantitative, multiplex, real-time fluorogenic PCR (TaqMan) assay. Appl. Environ. Microbiol. 66 2853-2858.

Wenneker, M., Verdel, M. S. W., Van Beuningen, A. R., Derks, J. H. J., and Janse, J. D. 1999. Ralstonia (Pseudomonas) solanacearum race 3 (biovar 2) in surface water and natural weed hosts: First report on stinging nettle (Urtica dioica). Eur J. Plant Pathol. 105:307-315.

Wicker, E., Grassart, L., Coranson-Beaudu, R., Mian, D., Guilbaud, C., Fegan, M., and Prior, P. 2007. Ralstonia solanacearum strains from Martinique (French west indies) exhibiting a new pathogenic potential. Appl. Environ. Microbiol. 73:6790-6801. 
Wicker, E., Lefeuvre, P., de Cambiaire, J. C., Lemaire, C., Poussier, S., and Prior, P. 2012. Contrasting recombination patterns and demographic histories of the plant pathogen Ralstonia solanacearum inferred from MLSA. ISME J. 6:961-974.

Yahiaoui, N., Chéron, J.-J., Jeetah, R., Benimadhu, S., Félicité, J., Cellier, G., Prior, P., Guérin, F., and Poussier, S. 2016. First report of Ralstonia pseudosolanacearum phylotype I causing bacterial wilt on Rodrigues Island, Indian Ocean. Plant Dis. 100:2522.
Yahiaoui, N., Chéron, J.-J., Ravelomanantsoa, S., Hamza, A. A., Petrousse, B. Jeetah, R., Jaufeerally-Fakim, Y., Félicité, J., Fillâtre, J., Hostachy, B., Guérin, F., Cellier, G., Prior, P., and Poussier, S. 2017. Genetic diversity of the Ralstonia solanacearum species complex in the southwest Indian Ocean islands. Front. Plant Sci. 8:2139.

Yik, C. P., Ong, A. K., and Ho, R. 1994. Characterization of Pseudomonas solanacearum strains from Singapore. Singap. J. Prim. Ind. 22:57-62. 